\title{
Using Shared Medical Appointments to Increase Access to Buprenorphine Treatment
}

\author{
David Roll, MD, Margaret Spottswood, MD, and Hsiang Huang, MD
}

Introduction: This study examines patient experiences with shared medical appointments for buprenorphine treatment at a safety net primary care clinic.

Methods: This is a cross-sectional observational study of 28 participants in a primary care buprenorphine shared medical appointments program.

Results: Participants reported appreciating the group visit format, gaining increased coping skills, and having more stable housing and less legal difficulty after starting the program.

Conclusion: The implementation of shared medical appointments for buprenorphine treatment benefits clinicians and patients. The nurse care manager and buprenorphine prescriber can efficiently attend to the clinical needs of multiple patients. (J Am Board Fam Med 2015;28:676-677.)

Keywords: Drug Abuse, Patient-centered Care, Substance Abuse

Outpatient buprenorphine opioid addiction treatment is an effective intervention in primary care settings; however, uptake in clinical practice has been limited. ${ }^{1}$ A recent study described the feasibility of integrated buprenorphine treatment via additional support from a nurse care manager. ${ }^{2}$ Shared medical appointments for chronic medical conditions have been studied in primary care settings. This study examines patient experiences with buprenorphine shared medical appointments at a safety net primary care clinic.

This article was externally peer reviewed.

Submitted 12 January 2015; revised 13 May 2015; accepted 18 May 2015.

From the Department of Internal Medicine, Cambridge Health Alliance, Harvard Medical School, Cambridge, MA (DR); the Department of Psychiatry, Cambridge Health Alliance, Harvard Medical School, Cambridge, MA (MS, $\mathrm{HH}$ ); and the Institute of Psychiatry \& LIM-23, Faculty of Medicine, University of São Paulo, São Paulo, Brazil $(\mathrm{HH})$.

Funding: The research was supported by a grant from the Innovation Fellows Program of the Harvard Medical School Center for Primary Care (Dr. David Roll) and from the Health Services Division of the National Institute of Mental Health (grant no. T32 MH20021-14, Dr. Hsiang Huang).

Conflict of interest: none declared.

Corresponding author: Hsiang Huang, MD, Department of Psychiatry, Harvard Medical School, 1493 Cambridge St., Cambridge, MA 02139 (E-mail: hhuang@cha.harvard.edu).

\section{Methods \\ Setting}

Revere Family Health Center (Revere, MA) is a primary care clinic of $\sim 7000$ low-income patients in a community with a high prevalence of opioid use disorder. Shared medical appointments are run by a primary care physician (DR) and a certified addictions nurse. Visits include patient check-ins and structured educational modules. Eight to 10 patients are seen during a 75-minute session with visits occurring 1 to 4 times monthly. New participants join and leave as they start therapy, move groups, or terminate therapy.

\section{Sample and Procedures}

Of 31 possible patients, 28 participated in the study (responses were de-identified). Patients were 20 to 60 years old (mean, 40.7 years), were mainly men (71\%), and 50\% were hepatitis C-positive; $68 \%$ endorsed a diagnosis of depression, $79 \%$ were diagnosed with anxiety disorder, and 39\% with bipolar disorder. Visit participation ranged from 1 to 46 months. Multiple-choice and open-response questions about visit format and respondents' health, mental health, sobriety, and life circumstances were measured. Patients were asked to report life circumstances before treatment and at the time of the survey. The institutional review board of Cambridge Health Alliance approved this study. 


\section{Results}

Over $88 \%$ reported liking visits; reasons were "group support" (82\%), "learning things from other people that help me" (82\%), and "others in the group have a positive influence on my sobriety" (75\%). Dissatisfaction included concerns about privacy $(25 \%)$. Among the participants, $79 \%$ thought the group sizes were "about right."

Patients identified important topics covered in the educational modules as anxiety management (61\%), pain management (50\%), nutrition (50\%), and relationships (39\%). Since starting treatment $60 \%$ reported learning more about hepatitis C, $43 \%$ reported receiving hepatitis vaccinations, and $36 \%$ reported having additional monitoring tests.

Of the $32 \%$ of patients reporting "unsuitable housing" (i.e., homeless, living with drug abusers) before treatment, $\sim 80 \%$ indicated housing improvement. About 35\% reported increased time spent working. Participation in outside recovery groups reportedly increased from $30 \%$ to $80 \%$. Nearly all patients who reported outstanding legal cases said they had resolved after starting treatment.

\section{Discussion}

This study explored experiences of opioid use disordered patients in primary care buprenorphine shared medical appointments. Most found the format highly acceptable. Members noted benefit from social support and increased involvement in Narcotics/Alcoholics Anonymous, demonstrating format compatibility.

A quarter of participants reported privacy concerns. Although patients sign a waiver to keep contents of the group visits confidential, the possibility of sharing information is real. However, the risk is not substantially different from that of other common group therapy models.

Members expressed a desire for the expansion of shared visits for others in need of opioid use disor- der treatment. Appointments scheduled outside working hours may increase the rate of participation and optimize functional status for these patients.

The implementation of buprenorphine shared medical appointments benefits both clinicians and patients. The nurse care manager and buprenorphine prescriber can efficiently attend to the clinical needs of multiple patients using this model. A recent study showed that only half of patients screening positive for substance use disorder were referred for addictions treatment, ${ }^{3}$ highlighting the need for such programs. Limitations for this crosssectional observational study include recall bias and social desirability bias, especially since the survey was administered at the time of visits.

\section{Conclusion}

Buprenorphine shared medical appointments for opioid use disorder were found to be highly acceptable. Modifiable factors for improvement include increased availability of groups outside working hours and expanding the range of educational modules (eg, pain, anxiety management, nutrition, relationships).

The authors thank Francyne Puopolo, RN, for her commitment to the buprenorphine program and Erin Bettendorf, MD, for her assistance in the development of the survey used in this study.

\section{References}

1. Netherland J, Botsko M, Egan JE, et al. Factors affecting willingness to provide buprenorphine treatment. J Subst Abuse Treat 2009;36:244-51.

2. Alford DP, LaBelle CT, Kretsch N, et al. Collaborative care of opioid-addicted patients in primary care using buprenorphine: five-year experience. Arch Intern Med 2011;171:425-31.

3. Chan YF, Huang H, Sieu N, Unutzer J. Substance screening and referral for substance abuse treatment in an integrated mental healthcare program. Psychiatr Serv 2013;64:88-90. 